\title{
MODELO FRACTAL DE SUBSTÂNCIAS HÚMICAS
}

\author{
FRACTAL MODEL OF HUMIC SUBSTANCES
}

\author{
Alessandro Costa da Silva ${ }^{1}$ Eduardo de Sá Mendonça ${ }^{2}$
}

\section{- REVISÃo BIBLIOGRÁFICA -}

\section{RESUMO}

A teoria fractal, por meio da determinação da dimensão fractal (D), tem sido considerada como uma alternativa para explicar a conformação de agregados moleculares. Sua utilização no estudo de substâncias húmicas (SH) reside na tentativa de descrever (representar) a estrutura ramificada ou a superfície rugosa e distorcida destas substâncias. A presença de um modelo fractal por sistemas naturais implica que este pode ser decomposto em partes, em que cada uma, subseqüentemente, é cópia do todo. Do ponto de vista experimental, a dimensão fractal de sistemas húmicos pode ser determinada a partir de técnicas como turbidimetria, raios $x$, espalhamento de neutrons, dentre outras. Este trabalho pretende facilitar o entendimento sobre a aplicação de fractais ao estudo conformacional de $\mathrm{SH}$, introduzindo conceitos e informações sobre o fundamento dos modelos fractais, bem como sobre o uso da técnica turbidimétrica na determinação do valor $D$.

Palavras-chave: substâncias húmicas, fractais, turbidimetria.

\section{SUMMARY}

Fractal theoria application by determination of fractal dimension has been considered an alternative tool to explain the conformation of molecular aggregates. Its utilization in the study of humic substances (HS) aims the attempt to describe the limbed structure or the rugous and distorced surface of these substances. The presence of fractal models indicates that the system may be decomposed in parts, each part being a copy of the whole. In the experimental point of view the fractals models of natural systems may be measured through techniques as turbidimetry, $x$ - ray and neutrons scattering. This paper seeks to facilitate the understanding on the application of the fractals in the conformational study of HS, supply information about fractal models foundation and use of the turbidimetry in the determination of fractal dimension.
Key words: humic substances, fractals, turbidimetry.

\section{INTRODUÇÃO}

Substâncias húmicas $\quad(\mathrm{SH})$ são polieletrólitos orgânicos naturais formados por fortes alterações da matéria orgânica, como a degradação de restos de plantas e animais (STEVENSON, 1994). São caracterizadas por serem misturas fisicoquimicamente heterogêneas, com ausência de repetição estrutural e falta de ordem a nível molecular (HAYES, 1989). Estudos espectromicroscópicos de SH revelam dependência entre sua composição estrutural e o tipo de material (solo e água), bem como de sua origem (MYNEMI, et al., 1999).

Diversas propriedades da matéria orgânica do solo, em particular das SH, depende das suas características macromoleculares. Essas macromoléculas freqüentemente atingem dimensões que permitem classifica-las como colóides, i. é, agregados formados de numerosos átomos ou moléculas (NOVOTNY \& MARTIN-NETO, 1999). O estado coloidal ou suspensão compreende sistemas contendo tanto moléculas grandes como pequenas partículas, intervalo de 1 a $1000 \mathrm{~nm}$ (SHAW, 1975). As suspensões coloidais são consideradas estáveis quando suas partículas estão dispersas e não ocorre sedimentação gravitacional mensurável num período de 2 a 24 horas (SPOSITO, 1989).

\footnotetext{
${ }^{1}$ Doutorando em Solos e Nutrição de Plantas, Universidade Federal de Viçosa (UFV). Av. PH Rolfs s/n, 36571000, Viçosa, MG. E-mail: ale@alunos.ufv.br.

${ }^{2}$ Professor do Departamento de Solos, UFV. Bolsista do CNPq. E-mail:esm@mail.ufv.br. Autor para correspondência Recebido para publicação em 11.07.00. Aprovado em 27.12.00
} 
As partículas húmicas apresentam uma variedade de formas e tamanhos, a qual implica porosidade e compactação variável de sua estrutura, tanto no estado sólido quanto coloidal (SWIFT, 1989). Essa variedade na sua estrutura macromolecular, que vai do formato esferoidal a filamentoso, depende das condições do meio como concentração da amostra, $\mathrm{pH}$ e força iônica do meio (GHOSH \& SCHNITZER, 1980).

Moléculas, quando em concentrações definidas, podem formar agregados organizados ou micelas. Neste caso, as cadeias hidrofóbicas se orientam para o interior da micela, deixando os grupos polares em contato com o meio aquoso, sendo que a forma das miscelas individuais varia com a concentração (SHAW, 1976). As micelas de menor grupos funcionais ácidos podem aproximar-se o suficiente para formar agregados de elevada massa molar (PICCOLO et al., 1996). Quando em solução, as $\mathrm{SH}$ formam agregados (micelas) que são mantidos por pontes de hidrogênio e interações hidrofóbicas (CONTE \& PICCOLO, 1999). Entretanto, ainda são modeladas como compostos macromoleculares de massa molar variável e comportamento conformacional semelhante a proteínas (PICCOLO, 1998).

Diferente de proteínas, puras, as SH são classificadas como macromoleculas polidispersas, ou seja, misturas de moléculas com diferente massa molar e, consequientemente, de tamanho variado. De acordo com ATKINS (1998), conhece-se apenas suas massas molares médias e, dependendo da técnica, esses valores podem ser expressos diferentemente, devido, à possibilidade de ocorrência de agregação entre partículas húmicas.

O grau de irregularidade e rugosidade da superfície exposta de macromoléculas húmicas tem importância nos processos biogeoquímicos, além de influenciar no número, tipo e disponibilidade de sítios reativos e, consequentemente, na sua interação com minerais e íons metálicos (STEVENSON, 1994).

Como os sistemas formados por partículas húmicas são caracterizados por uma natureza extremamente complexa e irregular, no que tange a sua organização molecular, morfologia, porosidade, superfície exposta e fenômenos de agregação e dispersão (RICE \& LIN, 1994), não podem ser descritos pela geometria euclidiana. Esses sistemas, como a maioria dos fenômenos naturais, apresentam um comportamento não-linear, gerando padrões altamente complexos (PIRES \& COSTA, 1992). Uma alternativa que minimiza as limitações presentes na geometria euclidiana (clássica) dos fenômenos irregulares da natureza é a geometria fractal que, ao contrário da euclidiana, não se baseia em objetos regulares, suaves e diferenciáveis (GUZMAN et al., 1993).

Cabe ressaltar que os sistemas/conjuntos na geometria euclidiana são descritos por equações algébricas, enquanto na geometria fractal são descritos por algorítimos recursivos. Conhecendo o "motivo" gerador de uma determinada estrutura pode-se, a partir daí, obter a estrutura completa (VICSEK, 1992). Os sistemas euclidianos também chamados não-fractais são aqueles ordenados e que seguem a dimensão euclidiana ou topológica $\left(\mathrm{D}_{\text {top }}\right)$, cujos valores, diferente da fractal, devem ser inteiros. Neste caso, $\mathrm{D}_{\text {top }}=$ zero indica um ponto; $\mathrm{D}_{\text {top }}=1$ uma curva; $\mathrm{D}_{\text {top }}=2$ uma superfície; e $\mathrm{D}_{\text {top }}=3$ um volume. Essa dimensão apresenta sérias limitações para a representação dos fenômenos caóticos da natureza, pois, na maioria das vezes, o valor de $\mathrm{D}_{\text {top }}$ tende a ser fracionário, indicando que o sistema é fractal e, com isso, segue a dimensão fractal (SENESI, 1994).

O aspecto mais básico de um objeto fractal talvez seja a sua dimensão. Um modo simples e intuitivo de associar uma dimensão a um dado conjunto é contar o número mínimo $\mathrm{N}(\varepsilon)$ de quadrículas, de lado $(\varepsilon)$, necessárias para cobrir completamente o conjunto. Esse número obedece a uma lei de potência: $\mathrm{N}(\varepsilon)=A \cdot \varepsilon^{-\mathrm{D}}$, que define e permite calcular o expoente $\mathrm{D}$, que é a dimensão de cobertura ou mais simplesmente a dimensão fractal (MANDELBROLT, 1983).

A geometria fractal, por meio da dimensão fractal $\mathrm{D}$, pode apresentar valores fracionários que dependem do sistema: $1<\mathrm{D}<2$ para uma curva, $2<\mathrm{D}<3$ para superfície e $2<\mathrm{D}<3$ para um 'sólido'. O valor D de um objeto é a medida do grau de irregularidade considerada em todas as escalas, relacionado à crescente medida do objeto à proporção que o dispositivo de medida se torna menor (VICSEK, 1992). Uma alta dimensão fractal significa que o objeto é mais irregular e, com isto, a medida estimada aumenta mais rapidamente. $\mathrm{Ou}$ seja, esse valor (D) é uma medida da proporção do espaço realmente ocupado por um sistema desordenado, podendo refletir a conformação das partículas que o constituem (HARRISON, 1992).

A teoria fractal foi introduzida na década de 70 pelo matemático Benôit Mandelbrot para designar objetos e estruturas complexas dotadas da propriedade de autossimilaridade (CHAVES, 1989). Estruturas autossimilares possuem detalhes como ramificações, poros ou rugosidades, em uma certa faixa de escala de comprimento, cuja forma é a mesma (estatisticamente) em cada escala de 
observação nessa faixa. Assim, se uma parte da estrutura for ampliada terá a mesma forma do todo (MANDELBROT, 1983). Já o termo 'fractais' são representações geométricas de sistemas desordenados cuja estrutura pode ser descrita por dimensões não-integrais (GUZMAN et al., 1993). A natureza não-integral destas dimensões, indicam que objetos fractais devem possuir detalhes estruturais que possam acomodar esta desordem (VICSEK, 1992).

Nos últimos anos, houve um grande interesse na aplicação da teoria fractal em diversos ramos da ciência (BARTON \& LA POINTE, 1995; VICSEK, 1992; HARRISON, 1992). Embora o uso de fractais na ciência do solo seja recente (BURROUGH, 1983), já existem vários trabalhos nos quais é aplicada a teoria dos fractais no estudo estrutural de substâncias húmicas (SILVA \& MENDONÇA, 2000; MYNEMI et al., 1999; SENESI, et al., 1997; RICE \& LIN, 1994; dentre outros).

Experimentos têm demonstrado que SH podem ser descritos como sistemas fractais, tanto no estado sólido ou dissolvido (ÖSTERBERG \& MORTENSEN, 1994) quanto no coloidal ou em suspensão (SENESI et al., 1996). Isto implica que sistemas fractais podem ser decompostos em partes, em que cada uma é "cópia" do "todo", encurtando diferentes fatores em diferentes direções (MANDELBROLT, 1983).

De acordo com HARRISON (1992), as estruturas fractais ramificadas (como uma árvore), porosas (como uma esponja) e fragmentadas (como uma rachadura) são ditas fractais de massa $\left(D_{m}\right)$, de poros $\left(D_{p}\right)$ e de superfície $\left(D_{s}\right)$, respectivamente. Em geral, os sistemas formados por partículas húmicas não são considerados estruturas porosas, pois o tamanho desses poros (da ordem de nanômetros) é muito pequeno quando comparado com a escala da superfície dessas partículas. Com isso, as partículas húmicas apresentam-se, somente, como $\mathrm{D}_{\mathrm{m}}$. Nesse caso, a massa é proporcional à área superficial ou como $\mathrm{D}_{\mathrm{s}}$, consiste de duas regiões não fractais separadas por uma superfície fractal (SENESI, 1994).

$\mathrm{Na}$ geração de um modelo fractal, a autosimilaridade ou invariância de escala, é investigada por meio de uma lei de potência da forma $p \propto v$, onde $p=$ propriedade; $v=$ variável; e $\Upsilon=$ expoente, que pode ser relacionado à dimensão fractal. Essa expressão garante que sobre uma faixa de valores da escala da variável independente $v$, de pelo menos uma ordem de grandeza, o expoente $\Upsilon$ (e portanto, o valor de 'D'), pode ser calculado diretamente dos dados experimentais a partir da inclinação da porção linear do gráfico $\log p$ vs $\log v$ (SENESI, 1994).
Para a determinação do valor ' $D$ ', a partir da expressão $p \propto v^{\Upsilon}$, é imprescindível conhecerem qual propriedade (física, química ou bioquímica) o sistema está inserido e que, por sua vez, poderá ser descrito como lei de potência. Medidas como a turbidimetria (SENESI et al., 1996), espalhamento de neutrons (ÖSTERBERG \& MORTENSEN, 1994), espalhamento de raios-X (RICE \& LIN, 1993), dentre outras, já foram aplicadas no estudo de fractais visando inferir sobre a conformação e fenômenos de agregação de partículas de $\mathrm{SH}$.

A lei de potência: $\tau \propto \lambda^{\beta}$ (em que $\tau$ é a turbidez, $\lambda$ é o comprimento de onda e $\beta$ é um expoente diretamente relacionado com D) é observada em sistemas de partículas em suspensão diluída, conforme demonstrado por meio de um modelo matemático descrito por HORNE (1987). Essa lei de potência, fundamentada na técnica turbidimétrica $(\tau)$, tem sido utilizada por pesquisadores (SILVA \& MENDONÇA, 2000 e SENESI et al., 1997) no estudo de SH. A utilização da técnica deve-se, principalmente, à rapidez e baixo custo (comparada com as demais técnicas), embora seu uso requeira que a suspensão contenha partículas monodispersas, de tamanho relativamente homogêneo e largas o suficiente para sofrer espalhamento independente. Ressalta-se que, para evitar o efeito do espalhamento múltiplo e interação entre partículas, o uso de suspensões diluídas também é requerido (HORNE, 1987).

Os fenômenos de espalhamento de luz e do movimento de partículas têm sido estudados desde as observações feitas por Robert Brown há quase dois séculos (SCHUKIN et al., 1998), sendo de grande valor na determinação do tamanho, forma e interação de partículas (SHAW, 1975). Quando um feixe de luz incide sobre uma amostra em suspensão, parte deste feixe atravessa a amostra e parte é refletido. As medidas em linha reta são denominadas turbidimétricas e aquelas em linha transversal $\left(I_{90} / I\right)$ são denominadas nefelométricas (EDWING, 1996).

A turbidimetria $(\tau)$, medida de redução na intensidade da luz incidente devido ao espalhamento, é definida como: $\tau=d$. $\ln$. (Io/I) onde $d$ é o comprimento óptico, $I o$ e $I$ são as intensidades incidente e transmitida, respectivamente. Nesse caso, a intensidade, polarização e distribuição angular da luz espalhada por uma dispersão coloidal dependem do tamanho e da forma das partículas, do espalhamento das interações entre essas partículas e da dispersão entre os índices de refração das partículas e o meio (SKOOG, et al., 1992). 
Nas partículas de peso molecular ' $M$ ', concentração ' $c$ ' e índice de refração ' $n$ ', o valor ' $\tau$ ' pode ser aproximado por: $\tau=H$. . $. M . Q$ [1], em que: $H$ é definido como $H=32 \pi^{2} n^{2}(d n / d c)^{2} / 3 N \lambda^{4}$, sendo $N=\mathrm{n}^{\mathrm{o}}$ de avogrado, $d n / d c=$ incremento do índice de refração específico, $\lambda=$ comprimento de onda; e $Q$ é o resultado da interferência interna de luz espalhada pela partícula em todos os ângulos (SENESI $\boldsymbol{e t}$ al., 1994).

Por meio da derivada da equação [1] e por rearranjos matemáticos, obtém-se a expressão: $\beta=4$ $-\gamma+d \log \tau / d \log \lambda$ ou $\beta=4,2+d \log \tau / d \log \lambda$ [2]. O valor de $\gamma(-0,2)$, normalmente usado para proteínas (HORNE , 1987), tem sido usado na obtenção do valor D de SH (RICE \& LIN, 1994). A analogia é justificada pelo fato do incremento do índice de refração específico de $\mathrm{SH}$ ser da mesma ordem de magnitude daqueles calculados para proteínas (SENESI et al., 1996). Assim, os valores de $\beta$, obtidos pela equação [2] ou experimentalmente pelo coeficiente angular da reta $(\log \tau$ vs $\log \lambda)$, indicarão a natureza do sistema: se $\beta \neq 3$ o sistema é desordenado, tem natureza fractal, se $\beta=3$ o sistema é ordenado e não é fractal. Ou seja, indicarão qual tipo de dimensão fractal o sistema apresenta: se $\beta<$ 3 implica a existência de $D_{m}$; nesse caso, $D_{m}=\beta$; se $3<\beta \leq 4$ implica $D_{s}$; nesse caso, $D_{s}=6-\beta$; e se $\beta \cong$ 3 implica que não terá natureza fractal, neste caso $\mathrm{D}$ $=\mathrm{D}_{\text {top }}($ SENESI, 1994).

A presença de colinearidade entre $\tau$ e $\lambda$ indicará lei de potência do tipo: $\tau \propto \lambda^{\beta}$ e, consequentemente, a possibilidade de se calcular a dimensão fractal do sistema (SENESI et al., 1997). A dependência de $\tau$ em função de $\lambda$ foi confirmada por SILVA \& MENDONÇA (2000) em suspensões de $\mathrm{AH}$ com diferentes valores de $\mathrm{pH}(3,0 ; 5,0$ e 7,0) e tempos de agitação $(1,3,8,12$ e $24 \mathrm{~h})$, sendo a linearidade considerada satisfatória $(R \geq 0,997)$. Assim, as partículas em estudo podem exibir estrutura fractal de massa ou de superfície, e os valores de $D_{m}$ e $D_{S}$ podem ser obtidos diretamente de $\beta$. Esses autores ressaltam que a faixa, no visível, de comprimento de onda usada (400 a 550nm) ainda é considerada pequena para se afirmar com certeza sobre a natureza fractal de $\mathrm{SH}$ e se determinar suas dimensões fractais com precisão. SENESI $\boldsymbol{e t}$ al. (1994) explicam que a utilização de uma pequena faixa de variação do $\lambda$ deve-se ao fato de valores acima de 550nm não apresentarem colinearidade entre $\tau$ e $\lambda$.

Ressalta-se que, para a comparação de resultados, é necessário que os procedimentos analíticos, como preparo de amostra, meio em que se encontra, $\mathrm{pH}$, temperatura e técnica utilizada sejam semelhantes. SENESI et al. (1997) utilizando UVvis, observaram uma leve diminuição nos valores de D em função do tempo de equilíbrio. Já ÖSTERBERG et al. (1994), trabalhando com espalhamento de neutrons, observaram um aumento nos valores de $\mathrm{D}$ com o aumento gradativo do tempo de equilíbrio. A explicação deve-se à força iônica do meio $\left(0,1\right.$ mo $\ell \ell^{-1}$ de $\left.\mathrm{Na} \mathrm{Cl}\right)$ e à alta concentração de SH em suspensão $\left(3 g \ell^{-1}\right)$ utilizada neste experimento, enquanto que para SENESI et al. (1997), não foi adicionado sal e a concentração de SH em suspensão foi de apenas $30 \mathrm{mg} \ell^{-1}$. Alguns autores (GHOSH \& SCHINITZER, 1980) observaram que quanto maior a força iônica e a concentração de SH no meio, mais enovelada estavam as moléculas destas substâncias.

No que diz respeito à temperatura durante o experimento, ÖSTERBERG \& MORTENESEN (1994) confirmaram variações nos valores de D em função da temperatura do meio, os autores observaram mudanças de 1,80 quando as supensões encontravam-se a $8^{\circ} \mathrm{C}$, para 2,35 a $21^{\circ} \mathrm{C}$, indicando que as partículas tornaram-se mais enoveladas com o aumento da temperatura. Tais resultados também foram confirmados por SILVA (informação pessoal).

SENESI et al. (1997) observaram que, para uma mesma amostra, em mesmo $\mathrm{pH}$, mesma força iônica e apenas os tempos de equilíbrio sendo diferentes, as partículas húmicas apresentam mudanças conformacionais tão expressivas que em alguns casos comportaram-se como sistemas fractais e outros como não fractais. Tal comportamento, também foi confirmado por SILVA \& MENDONÇA (2000). A explicação para essas variações são as modificações das macromoléculas húmicas no estado coloidal, ocorridas possivelmente por ionização de grupos ou formação de pontes de hidrogênio, decorrentes, por sua vez, de variações no pH do meio (SWIFT, 1989).

No que tange às mudanças conformacionais decorrentes da variação no $\mathrm{pH}$ do meio SCHNITZER (1978) observou uma transição gradual de estrutura fibrosa a baixos valores de $\mathrm{pH}$, para uma estrutura laminar a valores de $\mathrm{pH}$ maiores com redução simultânea no tamanho das partículas. SENESI et al. (1996) e SILVA et al (em publicação) observaram, em amostras de $\mathrm{AH}$ adquiridas comercialmente pela Sociedade Internacional de Substâncias Húmicas (IHSS) e pela Fukla S/A, respectivamente, uma diminuição da dimensão fractal dos $\mathrm{AH}$ (aumento da rugosidade, estrutura mais aplainada) com o aumento do $\mathrm{pH}$ e um aumento da dimensão (estrutura mais colapsada) a 
baixo pH. Esse comportamento, já esperado, é explicado devido a ligações de hidrogênio, forças de Van der Walls e interações entre elétrons $\pi$ de moléculas adjacentes que ocorreriam a baixos valores de $\mathrm{pH}$. Com o aumento do $\mathrm{pH}$, as partículas húmicas se expandiriam e sofreriam repulsão eletrostática, pois estas forças tornam-se mais fracas com a dissociação de grupos carboxílicos e fenólicos, gerando cargas negativas. De acordo com NOVOTNY \& MARTIN-NETO (1999), esse fenômeno também pode explicar a solubilidade de $\mathrm{AH}$ a diferentes valores de $\mathrm{pH}$, pois com a expansão da molécula causada pelo aumento do $\mathrm{pH}$, a sua solvatação é facilitada.

Embora a extração de $\mathrm{AH}$ com $\mathrm{NaOH}$ permita que grupos ácidos sejam dissociados, devido à repulsão de carga proporcionada pela elevação do $\mathrm{pH}$ e, com isto, tenha uma conformação expandida (baixos valores de D), este comportamento, em alguns casos, pode ser modificado. É sabido (STEVENSON, 1994) que mudanças conformacionais em partículas húmicas são dependentes de vários fatores como origem do material e processo de extração/purificação. E para um mesmo tipo de material, estas mudanças ainda dependerão das condições do meio: concentração das SH, força iônica e pH (CHEN \& SCHNITZER, 1989). SILVA et al. (em publicação) ratificaram esta dependência, pois observaram, em amostras de $\mathrm{AH}$ extraídos e purificados de solo (Espodossolo), um comportamento diferente daquele obtido para $\mathrm{AH}$ adquiridos, comercialmente, da Fukla. A diferença foi que a dimensão fractal aumentou (estrutura mais colapsada) com a elevação do $\mathrm{pH}$. Os autores explicaram que, mesmo utilizando a metodologia de fracionamento de SH segundo a IHSS (SWIFT, 1996), existem alguns procedimentos de purificação que são mais específicos para um determinado tipo de amostra. Com isso, podem-se induzir modificações das condições do meio e conseqüentemente mudanças conformacionais na molécula (SWIFT, 1985). Quando à purificação, isto é, à remoção de impurezas inorgânicas e orgânicas como carboidratos, lipídeos e compostos de baixo peso molecular, agressiva pode-se ainda estar "selecionando" aquele material húmico e consequentemente "degradando" a molécula.

\section{CONSIDERAÇÕES FINAIS}

Dentre as ferramentas usadas para se conhecer a estrutura e conformação de SH como IV (infra-vermelho), UV (ultra-violeta), RMN (ressonância magnética nuclear) e Raios-X, a teoria fractal aparece como um dos modelos mais adequados. A aplicação desta teoria ao estudo de SH visa a geração de dados para elaboração de um modelo matemático que represente suas moléculas, aparentemente, de natureza desordenada (RICE \& LIN, 1993). Este modelo, visualizado por meio da determinação da dimensão fractal (D), tenta representar a estrutura irregular, rugosa e distorcida das SH.

$\mathrm{O}$ valor $\mathrm{D}$, obtido por medidas indiretas (no caso a turbidimetria), é considerado eficiente para se inferir sobre conformação. Entretanto, algumas premissas são imprescindíveis para a confiabilidade do uso de um modelo fractal e para a comparação de resultados como: a) que as suspensões contenham partículas monodispersas e b) especificar as condições do meio e o tipo de amostra (origem, teor de cinzas, composição elementar, espectros de infravermelho, razão $\mathrm{E}_{4} / \mathrm{E}_{6}$, dentre outros).

Tudo indica que está surgindo uma nova ferramenta para compreender a conformação de partículas heterogêneas, bem como elucidação de questões abertas a respeito de gênese, natureza estrutural, reatividade superficial, fenômenos de agregação e dispersão de SH.

\section{REFERÊNCIAS BIBLIOGRÁFICAS}

ATKINS, P.W. Physical chemistry. 6 ed. Oxford : Oxford University, 1998. 1018p.

BARTON, C.C., LA PONTE, P.R., Fractais in the earth sciences. New York : Plennum, 1995. 265p.

BURROUGH, P.A. Multiscale source of spacial variation in soil. The aplication of fractal concepts to nested levels of soil variation. J Soil Sci., v.34, p.577-597, 1983.

CHAVES, C.M.G.F. Fenômenos de agregação. Ciência Hoje, v.10, p.27-32, 1989

CHEN, Y., SCHNITZER, M. Sizes and shapes of humic substances by electron microscopy (621-637). In: HAYES, M.H.B., MaCARTHY, P., MALCOLM, R.L., et al. Humic Substance II: In search of structure. New York : John Wiley \& Sons, 1989. 764p.

CONTE, P., PICCOLO, A. Conformational arrangement of dissolved humic substances. Influence of solution composition on association of humic molecules. Environ Sci Technol, v.33, p.1682-1690, 1999.

EDWING, G.W. Métodos instrumentais de análise química. São Paulo : Edgard Blucher, 1996. V. I. 296p.

GHOSH, K., SCHNITZER, M. Macromolecular structures of humic substances. Soil Sci., v.129, p.266-276, 1980.

GUZMAN. M., MARTIN, M.A., MORÁN, M., et al. Estructuras fractales y sus aplicaciones. Barcelona : Labor, 1993. 279p.

Ciência Rural, v. 31, n. 5, 2001. 
HARRISON, A. Fractals in chemistry. Oxford : Science Publications, 1992. 90p.

HAYES, M.H.B., MaCARTHY, P., MALCOLM, R.L., et al. Structures of humic substances: the emergence of forms (3-31). In: HAYES, M.H.B., MaCARTHY, P., MALCOLM, R.L., et al. Humic Substance II: In search of structure: setting the scene. New York : John Wiley \& Sons, 1989. 764p.

HORNE, D.S. Determination of the fractal dimension using turbidimetric techniques. Faraday Discuss Chem Soc, v.83, p.259-270, 1987.

MANDELBROT, B.B. The fractal geometry of nature update and augmented. New York : Freeman, 1983. 568p.

MYNEMI, S.C., BROWN, J.T., MARTINEZ, G.A, et al. Imaging of humic substances macromolecular structures in water and soils. Science.v.386, n.5443, p.1335-1337, 1999.

NOVOTNY, E.H., MARTIN-NETO, L. Propriedades coloidais da matéria orgânica (41-67) In: SANTOS, G.A., CAMARGO, F.A.O. (Eds.) Fundamentos da matéria orgânica do solo: Ecossistemas tropicais e subtropicais. Porto Alegre : Genesis, 1999. 491p.

ÖSTERBERG, R., MORTENSEN, K. Fractal geometry of humic acids, temperature-dependent restructuring studied by smallangle neutron scattering. (127-132). In: SENESI, N., MIANO, T.M. Humic substances in the global environment and implications on human health. Amsterdam : Elsevier Science, 1994. 1368p.

ÖSTERBERG, R., SZAJADAK, L., MORTENSEN, K. Temperature-dependent restruring of fractal humic acids: A proton-dependent process. Environ Int v.20, p.77-88, 1994.

PICCOLO, A. Hydrophobic interactions controlling molecular sizes of humic molecules in soils. Effects on the accumulation and decomposition of soil organic matter. In: WORLD CONGRESS OF SOIL SCIENCE, 16, Montpellier. Anais... Montpellier : International Soil Science Society, 1998. CDROM

PICCOLO, A., NARDI, S., CONCHERI, G. Micelle-like conformation of humic substances as revealed by size exclusion chromatography. Chemosphere, v.33, p.595-602, 1996.

PIRES, A S.T., COSTA, B.V.A. A desordem inevitável. Ciência Hoje, v.14, n.8, p.35-39, 1992

RICE, J.A., LIN, J.S. Fractal dimensions of humic materials (115-120). In: SENESI, N. e MIANO, T.M. Humic substances in the global environment and implications on human health. Amsterdam : Elsevier Science, 1994. $1368 \mathrm{p}$.

RICE, J.A., LIN, J.S. Fractal nature of humic materials. Enviro. Sci Tecnhol, v.27, p.13-414, 1993.

SCHNITZER, M. Humic substances: chemistry and reactions (1-64). In: SCHNITZER, M, KHAN, S.U. (Eds.) Soil organic matter. Amsterdam : Elsevier, 1978. 290p.
SCHUKIN, E.D., PERTSON, A.V., AMELINA, E.A. La química coloidal. Barcelona : M. Morcü, 1998. 383p.

SENESI, N. The fractal approach to the study of humic substances (3-41). In: SENESI, N., MIANO, T.M. Humic substances in the global environment and implications on human health. . Amsterdam : Elsevier Science, 1994. 1368p.

SENESI, N., LOURUSOO, G.F., MIANO, T.M., et al. The fractal dimension of humic substances as function of $\mathrm{pH}$ by turbidity measurements (121-126). In: SENESI, N., MIANO, T.M. Humic substances in the global environment $\mathrm{e}$ implications on human health. Amsterdam : Elsevier Science, 1994. 1368p.

SENESI, N., RIZZI, F.R., DELLINO, P., et al. Fractal dimension of humic acids in aqueous suspension as a function of $\mathrm{pH} \mathrm{e}$ time. Soil Sci Soc Am J, v.60, p.1773-1780, 1996.

SENESI, N., RIZZI, F.R., DELLINO, P., et al. Fractal humic acids in aqueous suspensions at various concentrations, ionic strengths, and $\mathrm{pH}$ values. Coll and Surf, v.127, p.57-68, 1997.

SHAW, D.J. Introdução à química dos colóides e superfície. São Paulo : Edgard Blucher, 1975. 185p.

SILVA, A.C., MENDONÇA, E.S. Dimensão fractal de ácidos húmicos por meio de turbidimetria. Rev Ceres v.47, n.269, p.103-112, 2000.

SILVA, A.C., MENDONÇA, E.S., MARTINS, M.L., et al. Natureza fractal de ácidos húmicos. Rev Bras Cie Solo. (Aceito para publicação em junho/2000).

SKOOG, D.A, HOLLER, F.J., NIEMAN, T.A Principles of instrumental analysis. 5. ed. Philadelphia : Saunders Golden, 1992. 849p.

SPOSITO, G. The chemistry of soils. New York : Oxford University, 1989. 272p.

STEVENSON, J.F. Humus chemistry - Genesis, composition, reactions. 2. ed. New York : John Wiley \& Sons, 1994. 496p.

SWIFT, R.S. Method for extraction of IHSS soil fulvic and humic acids. In: SPARK, K.M., WELLS, J.D., JOHNSON, B.B Methods of soil analysis. Madison : Soil Science of Society of Americam, 1996. p.1018-1020.

SWIFT, R.S. Molecular weight, size, shape, and charge characteristics of humic substances: some basic considerations (449-465). In: HAYES, M.H.B. Humic Substance II: In search of structure. New York : John Wiley \& Sons, 1989. 764p.

SWIFT, R.S. Fractionation of soil humic substances (387-408) In: AIKEN, G.R., MCKNIGHT, D.M., WERSHAW, R.L. Humic substances in soil, sediment and water: geochemistry, isolation and characterization. New York : John Wiley \& Sons, 1985. 692p.

VICSEK, T. Fractal growth fenomena. 2. ed. Singapore : World Scientic, 1992. 488p.

Ciência Rural, v. 31, n. 5, 2001. 